\title{
Zur Kenntniss des Anemonins
}

\author{
(II. Mittheilung über Anemonin)
}

von

\section{Dr. Hans Meyer.}

Aus dem chemischen Laboratorium der k. k. deutschen Universität in Prag.

(Vorgelegt in der Sitzung am 15. Juni 1899.)

In einer ersten Mittheilung über Anemonin ${ }^{1}$ habe ich die Vermuthung ausgesprochen, dass dieser Pflanzenstoff als Stammsubstanz des Cantharidins anzusprechen sei, und habe Versuche in Aussicht gestellt, welche dahin abzielen sollten, die möglicherweise nach dem Schema

$$
\mathrm{C}_{10} \mathrm{H}_{8} \mathrm{O}_{4}+4 \mathrm{H}=\mathrm{C}_{10} \mathrm{H}_{12} \mathrm{O}_{4}
$$

verlaufende Reaction zu realisiren.

Die Verfolgung dieses Planes nöthigte $\mathrm{zu}$ einer Revision der bislang geltenden Spiegel'schen Cantharidinformel, deren Unhaltbarkeit gezeigt, und die durch ein neues, allen Reactionen dieses Körpers Rechnung tragendes Configurationsschema ersetzt werden konnte. ${ }^{2}$

Da somit die Frage nach der Constitution des Cantharidins und seiner Isomeren bis zu einem gewissen Grade als gelöst betrachtet werden kann, erscheint es an der Zeit, die Untersuchung des Anemonins wieder aufzunehmen, und sollen die folgenden Zeilen die Richtung skizziren, in welcher die dies-

1 Monatshefte für Chemie, 17, 283.

2 Hans Meyer, Über das Cantharidin. Monatshefte für Chemie, 18, 393. - Die Isomerien des Cantharidins. Monatshefte für Chemie, 19, 707. 
bezüglichen Versuche unternommen werden, vor Allem aber auch ein Beitrag zur Kenntniss des

\section{Ersten Reductionsproductes des Anemonins}

gegeben werden.

Im Jahre 1878 hat M. Hanriot eine Studie über das Anemonin publicirt, in welcher er auch die Einwirkung von Reductionsmitteln auf diesen Pflanzenstoff behandelt. ${ }^{1}$

Während weder Zinkstaub, noch Jodwasserstoffsäure beim Anemonin selbst $z u$ einer glatten Reaction führten, der grösste Theil der Substanz vielmehr bei der erforderlichen hohen Temperatur zerstört wurde, gelang es Han rio t, ein Bromderivat zu erhalten, welches sich als der Reduction leicht zugänglich erwies.

Hanriot ertheilt seinem Tetrabromid die Formel $\mathrm{C}_{15} \mathrm{H}_{12} \mathrm{O}_{6} \mathrm{Br}_{4}$ und dem Anemonin selbst die entsprechende für $\mathrm{C}_{15}$ geltende Moleculargrösse.

Da seither von Bekurts ${ }^{2}$ und von mir $^{3}$ die Formel mit $C_{10}$ begründet worden war, erschien das mit letzteren Bestimmungen unvereinbare Resultat der Untersuchungen des französischen Forschers einer Überprüfung bedürftig.

Nach Han riot löst man den Pflanzenstoff in Chloroform, fügt Brom hinzu und lässt bis zur Entfärbung stehen. Nach dem Verjagen des Chloroforms soll dann das Tetrabromid krystallisirt zurückbleiben und wird durch Waschen mit Äther von gummiartigen Schmieren befreit.

Bei in verschiedener Weise modificirter Wiederholung dieses Versuches konnte ich ebensowenig wie bei genauer Befolgung der angegebenen Vorschrift den gesuchten Körper erhalten; die nach dem Waschen mit Äther zưrückbleibende Substanz erwies sich vielmehr als unverändertes Anemonin und die extrahirten, gelb bis braun gefärbten, unkrystallisirbaren Schmieren enthielten alles Brom.

Beispielsweise lieferten $0.5 \mathrm{~g}$ Anemonin, in angeführter Weise behandelt, nach entsprechender Reinigung mit kaltem

1 Bull. soc. chim. (II), 47, 683.

2 Arch. für Pharm., 230, 182 (1892).

3 Monatshefte für Chemie, 17, 283. 
Äther circa $0.3 g$ eines krystallisirten bromfreien Rückstandes, der bei $146-148^{\circ}$ schmolz und der Analyse nach aus Anemonin bestand.

$0.2014 \mathrm{~g}$ bei $100^{\circ}$ getrockneter Substanz gaben $0.458 g \mathrm{CO}_{2}$ und $0.0836 \mathrm{gH}_{2} \mathrm{O}$.

In 100 Theilen:

$\begin{array}{ccc}\text { C } \ldots \ldots \ldots & \begin{array}{c}\text { Berechnet für } \\ \mathrm{C}_{10} \mathrm{H}_{8} \mathrm{O}_{4}\end{array} & \frac{62 \cdot 5}{62 \cdot 02} \\ \mathrm{H} \ldots \ldots \ldots & 4 \cdot 17 & 4 \cdot 61\end{array}$

Das Tetrabromid von Hanriot scheint daher bis aut Weiteres aus der Literatur zu streichen, die von diesem Forscher analysirte Probe dürfte ungenügend gereinigtes Anemonin gewesen sein, das dann weiter zur Reduction benützt wurde.

Wirklich lässt sich reines Anemonin nach dem für das »Tetrabromid « angewandten Verfahren reduciren; während ich aber auch hier, die Zusammensetzung des Reductionsproductes betreffend, von Hanriot's Analysen abweichende Zahlen gewärtigen zu müssen glaubte, erhielt ich zu meinem grossen Erstaunen ein Product, das in seinen Eigenschaften und seiner empirischen Zusammensetzung mit dem von Hanriot beschriebenen Hydroanemonin $\mathrm{C}_{15} \mathrm{H}_{20} \mathrm{O}_{6} \mathrm{H}_{2} \mathrm{O}$ vollkommen übereinstimmte!

$3 \mathrm{~g}$ Anemonin wurden mit $100 \mathrm{~cm}^{3}$ Alkohol und ebensoviel concentrirter Salzsäure auf dem Wasserbade erhitzt und successive $15 \mathrm{~g}$ chemisch reines Zink in granulis eingetragen. Die Flüssigkeit wird nach und nach rothgelb. Man erhitzt weiter, bis die Färbung wieder verschwunden, unter steter Erneuerung von concentrirter Salzsäure und Alkohol. Nach etwa sechs Stunden ist die Reaction zu Ende.

Man filtrirt, verdünnt mit Wasser und schüttelt die milchig getrübte Flüssigkeit sechsmal mit je $50 \mathrm{~cm}^{3}$ Chloroform aus. Dasselbe hinterlässt einen schwach gelblich gefärbten Rückstand, der, anfänglich syrupös, nach dem Anrühren mit etwas leichtflüchtigem Ligroin und beim Stehen über Schwefelsäure im Vacuum nach einigen Tagen in eine farblose Krystallmasse 
übergeht, die durch Abpressen von einer öligen, unangenehm riechenden Beimengung getrennt wird.

Nach dem Umkrystallisiren aus Alkohol und aus Chloroform wurden feine farblose Nädelchen oder Plättchen erhalten, anscheinend einheitlich und fast unzersetzt flüchtig, aber von unscharfem Schmelzpunkte. Es wurden Fractionen vom Schmelzpunkte $51-78^{\circ}$ erhalten; eine Probe der Hauptfraction, die sich bei $74-76^{\circ}$ verflüssigte, wurde verbrannt.

$0.1886 g$ gaben $0.1180 g_{2} \mathrm{O}$ und $0.393 g \mathrm{CO}_{2}$.

In 100 Theilen:

$$
\begin{aligned}
& \text { Gefunden } \\
& \text { C..... } 56 \cdot 83 \\
& \text { H. .... } 6.95
\end{aligned}
$$

Hanriot hatte für sein Hýdroanemonin die ähnlichen Werthe

$$
\begin{array}{cr}
\text { C } \ldots \ldots \ldots & 57 \cdot 13 \\
\mathrm{H} \ldots \ldots \ldots & 7 \cdot 11
\end{array}
$$

erhalten und der bei $78^{\circ}$ schmelzenden Substanz die Formel

$$
\mathrm{C}_{15} \mathrm{H}_{20} \mathrm{O}_{6}, \mathrm{H}_{2} \mathrm{O}
$$

ertheilt, welche verlangt:

$$
\begin{array}{lr}
\text { C ...... } & 57 \cdot 32 \\
\text { H..... } & 7 \cdot 04
\end{array}
$$

Dieses befremdliche Resultat erschien nun vorerst ganz räthselhaft, zumal da auch die Titration der in Alkohollösung deutlich sauer reagirenden Substanz einen unerwarteten Werth lieferte.

$0.1563 \mathrm{~g}$ einer bei $70-73^{\circ}$ schmelzenden Probe wurden in neutralisirtem Alkohol gelöst und mit zehntelnormalem $\mathrm{KOH}$ titrirt. Zur Neutralisation wurden $0.033 g$ Kali verbraucht:

Daraus berechnet sich

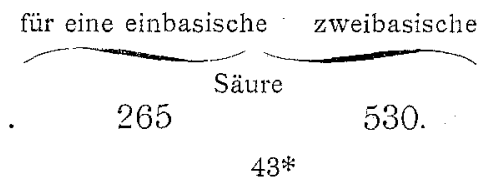

das Moleculargewicht ......... 
Sollte dem Anemonin doch die Formel $\mathrm{C}_{15} \mathrm{H}_{12} \mathrm{O}_{6}$ zukommen?

Dem widersprachen allerdings Beckurts und meine früheren Moleculargewichtsbestimnungen; es war aber immerhin möglich, dass mein vorliegendes Material, das ebenso wie das von Hanriot benützte aus Anemone pulsatilla bereitet worden war, mit der Substanz aus Anemone pratensis und Ranunculus sceleratus und acris, das zu meinen früheren Versuchen gedient hatte, nicht identisch und etwa polymer sei.

Es wurden daher mit dem Ausgangsmateriale einige Moleculargewichtsbestimmungen nach der Siedemethode ausgeführt.

Lösungsmittel: Benzol. Apparat von Beckmann.

\begin{tabular}{|c|c|c|c|c|c|c|}
\hline \multirow[b]{2}{*}{ 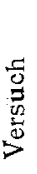 } & \multirow{2}{*}{$\begin{array}{c}\text { Gewicht der } \\
\text { Substanz } \\
\text { in Gramm }\end{array}$} & \multirow[b]{2}{*}{$\begin{array}{l}\text { Benzol } \\
\text { in Gramm }\end{array}$} & \multirow[b]{2}{*}{$\begin{array}{l}\text { Siedepunkt- } \\
\text { erhöhung }\end{array}$} & \multicolumn{3}{|c|}{ Moleculargewicht } \\
\hline & & & & gefunden & $\begin{array}{l}\text { berechnet } \\
\text { für } \\
\mathrm{C}_{10} \mathrm{H}_{8} \mathrm{O}_{4}\end{array}$ & $\begin{array}{l}\text { berechnet } \\
\text { für } \\
\mathrm{C}_{15} \mathrm{H}_{12} \mathrm{O}_{6}\end{array}$ \\
\hline 1 & $0 \cdot 1069$ & 1115 & $0 \cdot 150^{\circ} \mathrm{C}$ & 170 & & \\
\hline 2 & 2024 & $11 \cdot 15$ & 0.285 & 170 & 192 & 288 \\
\hline 3 & 3051 & $11 \cdot 15$ & 0.395 & 162 & & \\
\hline
\end{tabular}

Den Anemonin ist darnach endgiltig die Formel

zuzuweisen.

$$
\mathrm{C}_{10} \mathrm{H}_{8} \mathrm{O}_{4}
$$

Da nun bei der Reduction kaum eine Polymerisation stattgefunden haben konnte, da sich ausserdem ergab, dass beim wiederholten Umikrystallisiren der Substanz aus Wasser eine gewisse Veränderung derselben stattfand, insofern als dieselbe auf dem Wasserbade nur mehr theilweise verflüssigt wurde, so drängte sich die Vermuthung auf, dass der Alkohol sich an der Reaction betheiligt habe, das Reductionsproduct partiell in einen Ester verwandelt sei.

Damit harmonirte der negative Erfolg mehrfach unternommener Versuche, die Reduction des Anemonins mit wässeriger Salzsäure durchzuführen; in diesem Falle konnten stets nur geringe Mengen eines unkrystallisirbaren Syrups durch Chloroformextraction erhalten werden. 
Der sichere Beweis für die Annahme des Vorliegens einer äthoxylhaltigen Substanz wurde indessen durch eine Bestimmung nach dem Zeisel'schen Verfahren erbracht.

$0 \cdot 1887 \mathrm{~g}$ lieferten $0 \cdot 2165 \mathrm{~g}$ Jodsilber.

In 100 Theilen:

$$
\mathrm{C}_{2} \mathrm{H}_{5} \mathrm{O} \ldots \ldots \ldots \underbrace{\text { Gefunden }}_{22 \cdot 04}
$$

Anticipiren wir für den vollkommen alkylirten reducirten Ester die Formel

$$
\mathrm{C}_{10} \mathrm{H}_{12} \mathrm{O}_{5}\left(\mathrm{C}_{2} \mathrm{H}_{5}\right)_{2} \text {, }
$$

so ergibt sich der Estergehalt der untersuchten Probe zu $65 \%$.

Unter Benützung dieser Zahl und der aus der Titration gefundenen Moleculargrösse berechnet sich mit Hilfe der Gleichung

$$
65.270+35 x=100.265
$$

das Moleculargewicht des nicht äthylirten Reductionsproductes in erster Annäherung zu 250.

Wie später gezeigt werden wird, besitzt das Verseifungsproduct thatsächlich das Moleculargewicht 230 und die Formel $\mathrm{C}_{10} \mathrm{H}_{14} \mathrm{O}_{6}$.

Die zur Elementaranalyse verwendete Probe, welche, dem höheren Schmelzpunkte entsprechend, weniger Ester enthalten hat, bestand offenbar aus ungefähr gleichen Mengen Ester und freier Säure, wie aus der folgenden Zusammenstellung ersichtlich wird.

In 100 Theilen:

$$
\begin{aligned}
& \text { Berechnet für } \\
& \widehat{\mathrm{C}_{10} \mathrm{H}_{12} \mathrm{O}_{5}\left(\mathrm{C}_{2} \mathrm{H}_{5}\right)_{2}}-{\widehat{\mathrm{C}_{10} \mathrm{H}_{14} \mathrm{O}_{6}}}_{\text {Mittel }} \\
& \text { C...... } 62 \cdot 22 \\
& 52 \cdot 17 \quad 57 \cdot 2 \\
& \mathrm{H} \ldots . . .8 \cdot 14 \\
& 6 \cdot 09 \quad 7 \cdot 1
\end{aligned}
$$

Somit erscheinen auch Hanriot's Beobachtungen, soweit sie sein Hydroanemonin betreffen, in befriedigender Weise erklärt. 
Da durch fractionirtes Krystallisiren keine vollständige Trennung der beiden Bestandtheile zu erreichen war, wurde das Product verseift.

Die Substanz wurde mit sehr verdünnter Salzsäure unter Zusatz von Thierkohle einige Stunden gekocht, oder noch besser mit Wasser und Bromkohle.

Nach dem Filtriren und Concentriren erhält man dann feine farblose Krystallblättchen, die nach nochmaligem Umkrystallisiren aus Wasser vollkommen rein sind und den constanten Schmelzpunkt 151-153 zeigen. Die Elementaranalyse ergab mit der Formel $\mathrm{C}_{10} \mathrm{H}_{14} \mathrm{O}_{6}$ harmonirende Werthe.

I. $0 \cdot 2100 \mathrm{~g}$ gaben $0.1156 g \mathrm{H}_{2} \mathrm{O}$ und $0.3996 g \mathrm{CO}_{2}$.

II. $0 \cdot 1838 g$ gaben $0.1074 g \mathrm{H}_{2} \mathrm{O}$ und $0 \cdot 3520 \mathrm{~g} \mathrm{CO}_{2}$.

In 100 Theilen:

$$
\begin{aligned}
& \text { Berechnet für } \\
& \underbrace{\mathrm{C}_{10} \mathrm{H}_{14} \mathrm{~N}_{6}} \\
& \begin{array}{lll}
\text { C.... } 52 \cdot 17 \quad 51 \cdot 89 \quad 52 \cdot 23
\end{array}
\end{aligned}
$$

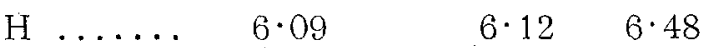

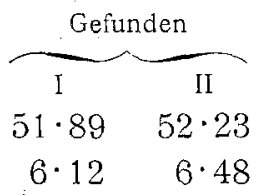

Dass die entstandene Substanz eine zweibasische Säure sei, liess sich dúrch Titration erweisen.

$0 \cdot 2644 \mathrm{~g}$, in Wasser gelöst und unter Verwendung von Phenolphtalein mit zehntelnormaler $\mathrm{KOH}$ versetzt, verbrauchten zur Neutralisation $0.1312 \mathrm{~g} \mathrm{KOH}$.

In 100 Theilen:

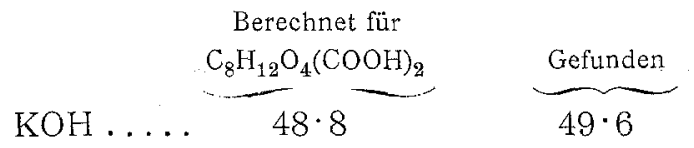

Daraus ergibt sich das

Moleculargewicht $\ldots . . \underbrace{\begin{array}{c}\text { Berechnet für } \\ \mathrm{C}_{10} \mathrm{H}_{14} \mathrm{O}_{6}\end{array}}_{230} \quad \frac{\text { Gefunden }_{226 \cdot 7}}{230}$

\section{Anemonolsäure $\mathrm{C}_{8} \mathrm{H}, \mathrm{G}(\mathrm{COOH})_{2}, \mathrm{H}_{2} \mathrm{O}$.}

Der so erhaltene Körper stellt offenbar die dem Anemonin entsprechende Oxysäure vor, und sei deswegen als Anemonolsäure bezeichnet. 
Wenngleich der Nachweis einer Hydroxylgruppe in derselben noch nicht mit wünschenswerther Schärfe direct durch Darstellung eines reinen Acetylderivates gebracht werden konnte, so ist doch das 'Verschwinden des Carbonylsauerstoffes bei der Aufnahme der beiden Wasserstoffatome sowohl aus der herabgeminderten. Empfindlichkeit der Substanz gegen Sodapermanganat, als auch vor Allem aus der Indifferenz der Anemonolsäure gegen Hydroxylamin, Phenylhydrazin in essigsaurer Lösung und Semicarbazid ersichtlich.

Die Anemonolsäure bildet in reinem Zustande prächtige farblose Plättchen von starkem Glasglanze, wenn sie aus Wasser, concentrisch gruppirte Nadeln; wenn sie aus verdünnter Salzsäure auskrystallisirt wurde. Sie löst sich leicht in heissem, mässig in kaltem Wasser, leichter in Alkohol, fast gar nicht in Äther. Chloroform und Essigäther nehmen sie in grosser Menge auf. Die Salze sind leicht löslich, mit Ausnahme der Silberverbindung.

\section{Anemonolsaures Silber.}

Wird eine mit Kalilauge vorsichtig neutralisirte wässerige Anemonolsäurelösung mit Silbernitrat versetzt, so fällt ein voluminöser farbloser Niederschlag der Silberverbindung aus.

Das Salz bildet nach dem Trocknen im Vacuum ein weisses glänzendes Pulver, das ziemlich lichtbeständig ist. Mit trockenem Äther oder Methylalkohol überschichtet, reagirt die Substanz schon bei Zimmertemperatur mit Jodmethyl; nach zwölfstündigem Stehen filtrirt man vom gebildeten Jodsilber $a b$ und verjagt das Lösungsmittel durch Erhitzen auf dem Wasserbade.

Der restirende, fast farblose Syrup erstarrt in der Kälte binnen Kurzem zu einer strahligen Krystallmasse, die abgepresst und aus Äther umkrystallisirt wird.

Die so erhaltene

\section{Dimethylanemonolsäure}

bildet feine weisse Nädelchen vom Schmelzpunkte $93-94^{\circ}$. Es ist leicht löslich in Alkohol und Äther. Wasser löst beim Kochen, aber die nach dem Erkalten ausfallenden Nädelchen 
sind grösstentheils verseift, was der bei $138-140^{\circ}$ liegende Schmelzpunkt der Hauptfraction und die stark saure Reaction der Lösung anzeigen.

Aus Essigäther krystallisirt der Ester sehr hübsch in grossen perlmutterglänzenden Plättchen. Der Schmelzpunkt zieht sich unscharf von $94-97^{\circ}$.

$0.228 \mathrm{~g}$ lieferten bei der Methoxylbestimmung $0.3554 \mathrm{~g}$ Jodsilber.

In 100 Theilen:

$$
\begin{aligned}
& \text { Berechnet für }
\end{aligned}
$$

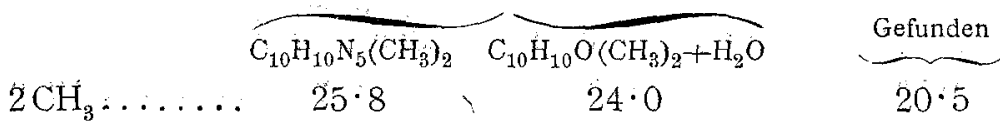

Trotz des schönen Aussehens war also auch dieses Produet einigermassen verseift. Wie auch in vielen anderen Fällen geht hier die leichte Verseifbarkeit mit der leichten Esterificirbarkeit Hand in Hand; es wird der Anemonolsäureester in fast quantitativer Weise bei der Reduction des Anemonins mit Zink und wässerig-methylalkoholischer Salżsäure gebildet.

Die Darstellung gelingt leicht in der beim Äthylderivate beschriebenen Weise. Der entstehende, fast farblose Syrup wird nach dem Impfen mit einer Spur Dimethylanemonolsäure fast vollständig zum Erstarren gebracht.

Das nicht an der Hydratbildung betheiligte Wassermolekül der Anemonolsäure ist ausserordentlich fest gebunden; so dass die Vermuthung gerechtfertigt erscheint, dass dasselbe als Constitutionswasser zu betrachten sei.

Das gesammelte Beobachtungsmaterial ermöglicht bereits jetzt, eine ziemlich gut definirte Structurformel für das Anemonin aufzustellen. In meiner ersten Abhandlung habe ich ausführlich dargelegt, dass die Carbonylgrủippe des Anemonins weder in $\alpha$-, noch $\beta$ - oder $\gamma$-Stellung zu einem der beiden anhydrisirten Carboxyle sich befinden kann. Vielmehr bildet die Ketongruppe einen Bestandtheil eines volikommen hydrirten 
Ringes; während die Carboxylgruppen einem zweiten Ringe angegliedert sind.

Das Anemonin liefert nun bei der Oxydation neben Oxalsäure ausschliesslich Bernsteinsäure in einer Menge vơn über $25 \%$; es enthält daher offenbar die Gruppirung:

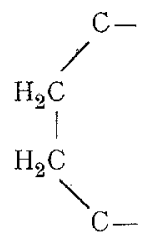

und zwar in jenem Kerne, welcher die Ketongruppe enthält. Die Analogie mit dem Cantharidin lässt uns in diesem Kerne einen Hexenonring vermuthen, der mit einem Tetramethylenringe in. Metastellung verbunden ist. An Letzterem befinden sich die Carboxyle analog wie im Isocantharidin:

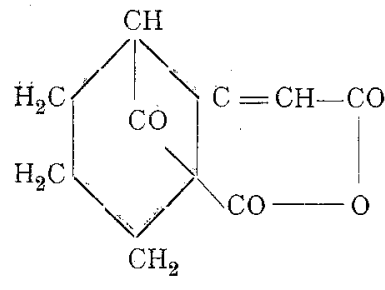

Anemonin

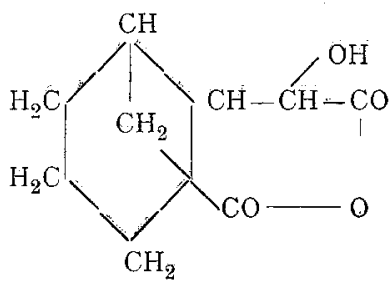

Isocantharidin.

Diese Formel ist geeignet, die merkwürdige Eigenthümlichkeit der neutralen Anemoninester zu erklären, mit sauren und alkalischen Reagentien verschiedene Verseifungsproducte zu liefern.

Wird z. B. Dimethylanemonin mit Alkali digerirt, so bildet sich die gelbe amorphe Anemoninsäure:

$$
\mathrm{C}_{8} \mathrm{H}_{8} \mathrm{O} \underset{\mathrm{H}}{\mathrm{OH}}(\mathrm{COOH})_{2},
$$

während die Verseifung durch Salzsäure zur Anemoninsäure:

führt.

$$
\mathrm{C}_{8} \mathrm{H}_{8} \mathrm{O}(\mathrm{COOH})_{2}
$$


Letztere Verbindung hat Bekurts auch durch Zersetzen des Anemoninbleis mit Schwefelwasserstoff erhalten. Da noch keine Elementaranalyse des Körpers vorliegt, seien die Resultate einer Verbrennung mit Bleichromat angeführt.

$0.1915 \mathrm{~g}$ gaben $0.0822 \mathrm{~g} \mathrm{H}_{2} \mathrm{O}$ und $0.4007 \mathrm{~g} \mathrm{CO}_{2}$.

In 100 Theilen:

$$
\begin{array}{ccc}
\mathrm{C} \ldots \ldots \ldots & \frac{\begin{array}{c}
\text { Berechnet für } \\
\mathrm{C}_{10} \mathrm{H}_{10} \mathrm{O}_{5}
\end{array}}{57 \cdot 14} \quad \underbrace{\text { Gefunden }} \\
\mathrm{H} \ldots \ldots \ldots & 4.76 & 5.07 \\
& 5.34
\end{array}
$$

Die Anemoninsäure bildet also eine Hydratform des Anemonins, aber nach der Definition von Hantzsch ${ }^{4}$ ein »abnormales Hydrat «, welches sich aus der wasserfreien Substanz nicht durch directe Wasseraufnahme, sondern nur dann bildet, wenn die Letztere vorher chemisch verändert worden war:

Auch ist, wie ich schon in meiner ersten Mittheilung erwähnte, eine Rückverwandlung in Anemonin aus der Anemoninsäure nicht durchführbar.

Dagegen liefert die Säure mit Kali und Jodäthyl leicht und quantitativ Diäthylanemonin, während ich mich seinerzeit vergeblich bemüht habe, die Äthylirung des Anemonins za einer vollständigen $\mathrm{zu}$ gestalten; es bilden sich hiebei immer gefärbte amorphe Nebenproducte in Menge und manchmal fast ausschliesslich.

Betrachten wir uns die Constitutionsformel des Anemonins, wie sie oben angeführt wurde, so erscheinen die beschriebenen Beobachtungen leicht verständlich.

Das Anemonin muss zwei Reihen von Derivaten zu geben befähigt sein, fumaroide:

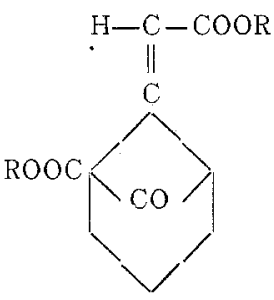

1 Berl. Ber., 32, 589. 
und maleïnoide:

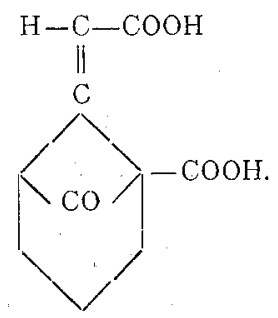

Das Anemonin selbst bildet das maleïnoide Anhydrid.

Beim Alkyliren bildet sich ein Gemenge der beiden möglichen neutralen Ester, von denen der stabile Cis-transester isolirt wurde und bei der Verseifung mit Säuren die Anemonsäure

liefert.

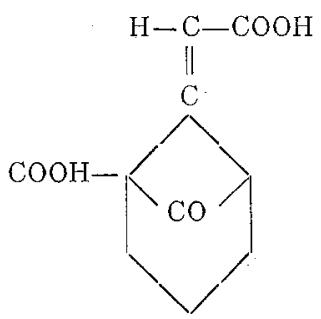

Bei der Einwirkung von Alkalien sowohl auf die Ester, wie auch auf das Anemonin selbst, wird Wasser an die doppelte Bindung addirt.

Bei der nun eintretenden Hydratisirung ist dann natürlich keine Isomerie mehr möglich; die Anemoninsäure erhält die Formel:

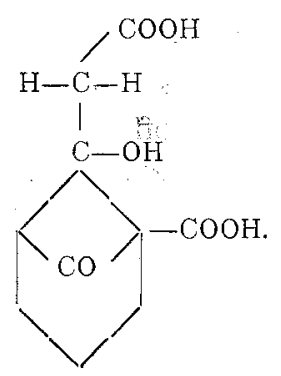

Nun erhält auch die gelbe Färbung dieser Säure und ihrer Derivate eine befriedigende Erklärung: Das eintretende 
Hydroxyl wirkt als Auxochrom der chromophoren Carboxylgruppe.

Wie ich seinerzeit erwähnt habe, kann der fast farblose Dimethylester des Anemonins auch in einer niedriger schmelzenden Form erhalten werden, welche ein Molekül Wasser mehr enthält und gelb gefärbt ist. ${ }^{1}$

Mit dem Verschwinden der chromophoren Gruppe verliert sich auch die Färbung; so ist die farblose Anemonolsäure:

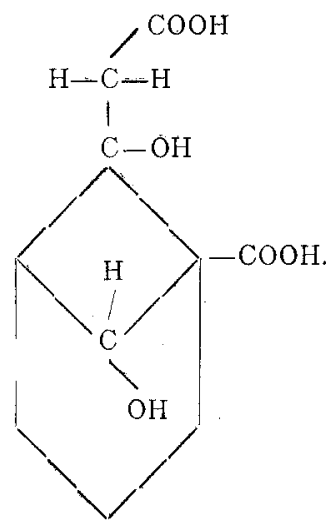

auch in Alkalien farblos löslich.

Mit der umfassenden experimentellen Prüfung der skizzirten Formel bin ich beschäftigt, und hoffe mir zur nächsten Blüthezeit der Anemonen die erforderliche Quantität des Ausgangsmaterials verschaffen zu können.

Inzwischen sei nur noch auf einen Punkt hingewiesen.

Hanriot hat bei der Zinkstaubdestillation des Anemonins in geringer Menge Kohlenwasserstoff erhalten, der gegen $150^{\circ}$ siedete und $89 \cdot 7 \%$ Kohlenstoff neben $11 \%$ Wasserstoff ergab. Es sind dies annähernd die Constanten des Cantharens.

1 S. 288. 